\title{
Slip analysis of magnetohydrodynamics flow of an upper-convected Maxwell viscoelastic nanofluid in a permeable channel embedded in a porous medium
}

\begin{abstract}
The continuous applications of viscoelastic fluids in biomedical engineering and industrial processes require some studies that provide better physical insights into the flow phenomena of the fluids. In this work, homotopy perturbation method is applied to investigate the simultaneous effects of slip and magnetic field on the flow of an upper convected Maxwell nanofluid through a permeable microchannel embedded in a porous medium. The results of the approximate analytical solution depict very good agreements with the results of the fourth order Runge-Kutta Fehlberg numerical method for the verification of the mathematical method used in analyzing the flow. Thereafter, the obtained analytical solutions are used to investigate the effects of pertinent rheological parameters on the flow process. It is observed from the results that increase in slip parameter, nanoparticle concentration and Darcy number lead to increase in the velocity of the upper-convected Maxwell fluid. However, when the Deborah's number increases, the Hartmann, and Reynold numbers decrease the fluid flow velocity towards the lower plate but as the upper plate is approached, a reverse trend is observed. The study can be used to advance the application of upper convected Maxwell flow in the areas of in biomedical, geophysical and astrophysics.
\end{abstract}

Keywords: slip analysis, upper-convected Maxwell flow, viscoelastic nanofluid, magnetic field, porous medium
Volume 2 Issue 5 - 2018

\author{
MG Sobamowo, AA Yinusa, AA Oluwo, SI \\ Alozie \\ Department of Mechanical Engineering, University of Lagos, \\ Nigeria
}

Correspondence: MG Sobamowo, Department of Mechanical Engineering, University of Lagos, Akoka, Lagos, Nigeria, Tel 2347034717417, Email mikygbeminiyi@gmail.com

Received:September 12, 2018 | Published: October II, 2018

\section{Introduction}

There have been increasing research works in the past few years on analysis of flow of viscoelastic fluid. This is due to its various applications in gaseous diffusion, blood flow through oxygenators, flow in blood capillaries have continue to aroused the research interests. However, complex rheological fluids such as blood, paints, synovial fluid, saliva, jam which cannot be adequately described by Navier Stokes. Consequently, complex constitutive relations that capture the flow behaviour of the complex fluids have been developed. ${ }^{1}$ Among the newly developed integral and differential-type fluid flow models, Upper convected Maxwell fluid model has showed to be an effective fluid model that captures the complex flow phenomena of fluids especially of the fluid with high elastic behaviours such as polymer melts. Such highly elastic fluids have high Deborah number. ${ }^{2-3}$ In the analysis of Maxwell flow, Fetecau ${ }^{4}$ presented a new exact solution for the flow of fluid through infinite microchannel while Hunt ${ }^{5}$ studied convective fluid flow through rectangular duct. Sheikholeslami et al. ${ }^{6}$ investigated magneto hydrodynamic field effect on flow through semi-porous channel utilizing analytical methods. Shortly after, Sheikholeslami ${ }^{7-9}$ adopted numerical solutions in the investigations of nanofluid in semi-annulus enclosure.

Flow of upper convected Maxwell fluid through porous stretch sheet was investigated by Raftari \& Yildirim. ${ }^{10}$ Entophy generation in fluid in the presence of magnetic field was analyzed by Sheikholeslami \& Ganji ${ }^{11}$ using lattice Boltzmann method while Ganji et al. ${ }^{12}$ explored analytical and numerical methods to analyze the fluid flow problems under the influence of magnetic field. The flow of viscoelastic fluid through a moving plate was analyzed by Sadeghy \& Sharifi ${ }^{13}$ using local similarity solutions. Vajrevulu et al. ${ }^{14}$ investigated the mass transfer and flow of chemically reactive upper convected Maxwell fluid under induced magnetic field. Not long after Raftari \& Vajrevulu ${ }^{15}$ adopted the homotopy analysis method in the study of flow and heat transfer in stretching wall channels considering MHD. Hatami et al. ${ }^{16}$ presented forced convective MHD nanofluid flow conveyed through horizontal parallel plates. Laminar thermal boundary flow layer over flat plate considering convective fluid surface was analyzed by Aziz ${ }^{17}$ using similarity solution. Beg $\&$ Makinde $^{18}$ examined the flow of viscoelastic fluid through Darcian microchannel with high permeability.

Most of the above reviews studies focused on the analysis of fluid flow under no slip condition. However, such an assumption of no slip condition does not hold in a flow system with small size characteristics size or low flow pressure. The pioneer work of flow with slip boundary condition was first initiated by Navier. ${ }^{19}$ The slip conditions occur in various flows such as nanofluids, polymeric liquids, fluids containing concentrated suspensions, flow on multiple interfaces, thin film problems and rarefied fluid problems. ${ }^{19-31}$ Due to the practical implications of the condition on the flow processes, several studies on the effects of slip boundary conditions on fluids flow behaviours have been presented by many researchers. ${ }^{19-35}$ Abbasi et al. ${ }^{36}$ investigated the MHD flow characteristics of upperconvected Maxwell viscoelastic flow in a permeable channel under slip conditions. However, an analytical study on simultaneous effects of slip, magnetic field, nanoparticle and porous medium on the flow characteristics of an upper-convected Maxwell viscoelastic nanofluid 
has not been carried out in literature. Therefore, in this work, homotopy perturbation method is used to analyze the slip flow of an upper-convected Maxwell viscoelastic nanofluid through a permeable microchannel embedded in porous medium under the influence of magnetic field is analyzed. Also, the effects of other pertinent parameters of the flow process are investigated and discussed.

\section{Model development and analytical solution}

Consider a laminar slip flow of an electrically conducting fluid in a microchannel is considered. Along the y axis, magnetic fields are imposed uniformly, as described in the physical model diagram Figure 1. It is assumed external electric field is zero and constant of electrical conductivity is constant. Therefore, magnetic Reynolds number is small and magnetic field induced by fluid motion is negligible.

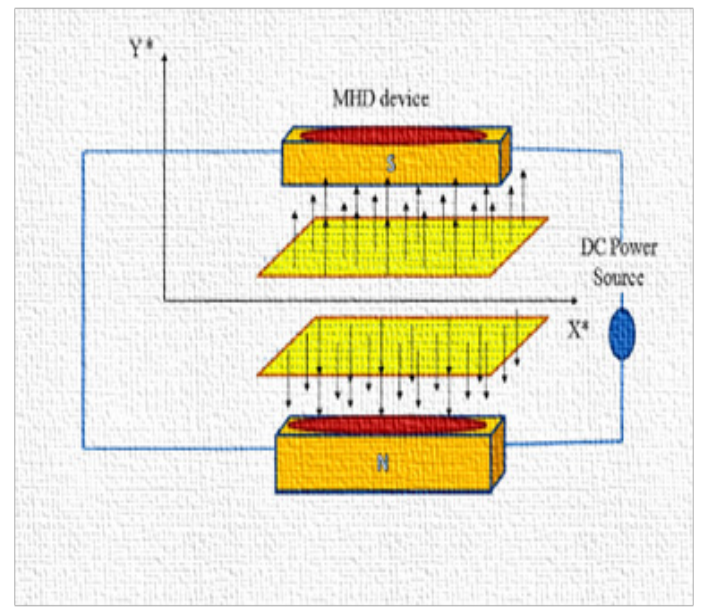

Figure I Flow of upper-convected Maxwell fluid between in permeable channel embedded in porous medium.

Based on the assumptions, the governing equation for the Maxwell fluid is presented as ${ }^{8}$

$$
\mathbf{T}=-P \mathbf{I}+\mathbf{S}
$$

where the Cauchy stress tensor is $\mathrm{T}$ and $\mathrm{S}$ is the extra-stress Tensor which satisfies

$$
\mathbf{S}+\lambda\left(\frac{\partial \mathbf{S}}{\partial t}+(\mathbf{V} \cdot \nabla) \mathbf{S}-\mathbf{L S}-\mathbf{S L}^{T}\right)=\mu A_{L}
$$

The Rivlin-Ericksen tensor is defined by

$$
A_{L}=\nabla V+(\nabla V)^{T}
$$

The continuity and momentum equations for steady, incompressible two dimensional flows are expressed in Eqs. (4) -(6) as

$$
\frac{\partial \bar{u}}{\partial x}+\frac{\partial \bar{v}}{\partial y}=0
$$

$$
\begin{aligned}
& \rho_{n f}\left(u \frac{\partial u}{\partial x}+v \frac{\partial u}{\partial y}\right)=-\frac{\partial P}{\partial x}+\frac{\partial S_{x x}}{\partial x}+\frac{\partial S_{x y}}{\partial y}-\sigma_{n f} B^{2}(t) u-\frac{\mu_{n f} u}{K_{p}} \\
& \rho_{n f}\left(\bar{u} \frac{\partial \bar{v}}{\partial x}+\bar{v} \frac{\partial \bar{v}}{\partial y}\right)=-\frac{\partial P}{\partial x}+\frac{\partial S_{y x}}{\partial x}+\frac{\partial S_{y y}}{\partial y}-\frac{\mu_{n f} \bar{v}}{K_{p}}
\end{aligned}
$$

where the effective density $\rho n f$ and effective dynamic viscosity $\mu_{n f}$ of the nanofluid are defined as follows:

$$
\begin{aligned}
& \rho_{n f}=(1-\phi) \rho_{f}+\phi \rho_{s}, \\
& \mu_{n f}=\frac{\mu_{f}}{(1-\phi)^{2.5}} \text {, } \\
& \sigma_{n f}=\sigma_{f}\left[1+\frac{3\left\{\frac{\sigma_{s}}{\sigma_{f}}-1\right\} \phi}{\left\{\frac{\sigma_{s}}{\sigma_{f}}+2\right\} \phi-\left\{\frac{\sigma_{s}}{\sigma_{f}}-1\right\} \phi}\right],
\end{aligned}
$$

and

$\mathrm{S}_{\mathrm{xx}}, \mathrm{S}_{\mathrm{xy}}, \mathrm{S}_{\mathrm{yx}}$ and $\mathrm{S}_{\mathrm{yy}}$ are extra stress tensors and $\rho$ is the density of the fluid. Using the shear-stress strain for a upper-convected liquid, The governing equations of fluid motion is easily expressed as 16

$$
\frac{\partial u}{\partial x}+\frac{\partial v}{\partial y}=0
$$

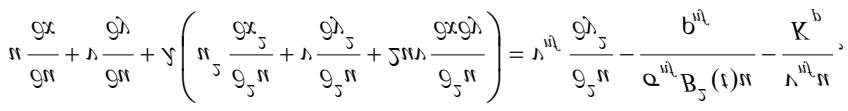

where flow velocity component $(u, v)$ are velocity component along the $\mathrm{x}$ and $\mathrm{y}$ directions respectively. Since flow is symmetric about channel center line, attention is given to the flow region $0<y<H$. Appropriate boundary conditions are given as

$$
\begin{aligned}
& y=0: \frac{\partial u}{\partial x}=0, v=0 \\
& y=H: \frac{\partial u}{\partial y}=-\beta u, \quad v=V_{w}
\end{aligned}
$$

where $V_{w}$ and $\beta$ are the wall characteristic suction velocity and sliding friction respectively. 
The physical and thermal properties of the base fluid and nanoparticles are given in Table 1 and Table 2, respectively.

The similarity variables are introduced as:

$$
\begin{aligned}
& \eta=\frac{y}{H}, \quad u=-V_{w} x f^{\prime}(y) ; \quad v=V_{w} f(y) ; \quad k=\frac{\mu}{H \beta} \\
& f^{\prime \prime \prime}-\left(M^{2}+\frac{1}{D a}\right) f^{\prime}+(1-\phi)^{2.5}\left((1-\phi)+\phi \frac{\rho_{s}}{\rho_{f}}\right) R e_{w}\left(f^{\prime 2}-f f^{\prime \prime}\right)+D e(1-\phi)^{2.5}\left(2 f f^{\prime} f^{\prime \prime}-f^{2} f^{\prime \prime \prime}\right)=0
\end{aligned}
$$

Table I Physical and thermal properties of the base fluid

\begin{tabular}{lllll}
\hline Base fluid & $\boldsymbol{\rho ( \mathrm { kg } / \mathbf { m } ^ { 3 } )}$ & $\boldsymbol{C}_{\boldsymbol{p}}(\mathrm{J} / \mathbf{k g K})$ & $\boldsymbol{k}(\mathrm{W} / \mathbf{m K})$ & $\boldsymbol{\sigma}\left(\Omega^{-1} \mathbf{m}^{-1}\right)$ \\
\hline Pure water & 997.1 & 4179 & 0.613 & 5.5 \\
Ethylene Glycol & 1115 & 2430 & 0.253 & 1.07 \\
Engine oil & 884 & 1910 & 0.144 & 4.02 \\
Kerosene & 783 & 2010 & 0.145 & 4.01 \\
\hline
\end{tabular}

Table 2 Physical and thermal properties of nanoparticles

\begin{tabular}{lllll}
\hline Nanoparticles & $\boldsymbol{\rho}\left(\mathbf{k g} / \mathbf{m}^{3}\right)$ & $\boldsymbol{C}_{p}(\mathrm{~J} / \mathbf{k g K})$ & $\mathbf{k}(\mathbf{W} / \mathbf{m K})$ & $\boldsymbol{\sigma}\left(\Omega^{-1} \mathbf{m}^{-1}\right)$ \\
\hline Copper $(\mathrm{Cu})$ & 8933 & 385 & 401 & 59.6 \\
Aluminum oxide $\left(\mathrm{Al}_{2} \mathrm{O}_{3}\right)$ & 3970 & 765 & 40 & 16.7 \\
SWCNTs & 2600 & 42.5 & 6600 & 1.26 \\
Silver $(\mathrm{Ag})$ & 10500 & 235 & 429 & \\
Titanium dioxide $\left(\mathrm{TiO}_{2}\right)$ & 4250 & 686.2 & 8.9538 & \\
Copper $(\mathrm{II})$ Oxide $(\mathrm{CuO})$ & 783 & 540 & 18 & \\
\hline
\end{tabular}

Taking boundary condition as

$$
\begin{aligned}
& \eta=0: f^{\prime \prime}=0 ; f=0 \\
& \eta=1: f^{\prime}=-k f^{\prime \prime}: f=1
\end{aligned}
$$

where $\operatorname{Re}_{w}=\frac{V_{w} H}{v}$ is the Reynolds number, $D e=\frac{\lambda V_{w}^{2}}{v}$ is the Deborah's number, $M^{2}=\frac{\sigma B_{0}^{2} H}{\mu}$ is the Hartman parameter,
$D a=\frac{K_{p}}{H}$ is the Darcy's number. For $\operatorname{Re}_{\mathrm{w}}>0$ corresponds to suction flow while $\operatorname{Re}_{\mathrm{w}}<0$ correspond to injection flow respectively.

Equ. (13) is a third-order differential equation with four boundary conditions. Through a creative differentiation of Eq. (12). Hence introducing fourth order equation as:

$$
f^{i v}-\left(M^{2}+\frac{1}{D a}\right) f^{\prime \prime}+(1-\phi)^{2.5}\left((1-\phi)+\phi \frac{\rho_{s}}{\rho_{f}}\right) R e_{w}\left(f f^{\prime \prime}-f f^{\prime \prime \prime}\right)+D e(1-\phi)^{2.5}\left(2 f^{\prime 2} f^{\prime \prime}-2 f f^{\prime \prime 2}+f^{2} f^{i v}\right)=0
$$

The above Eq. (14) study satisfies all the four boundary conditions

\section{Principles of homotopy perturbation method}

The following equation is considered in explaining the fundamentals of the homotopy perturbation method [10]

$$
A(u)-f(r)=0^{B\left(u, \frac{v u}{\partial \eta}\right)=0}
$$

Utilizing the boundary condition 


$$
B\left(u, \frac{\partial u}{\partial \eta}\right)=0 \quad r \in \Gamma
$$

A is the general differential operator, B is the boundary operator, $\mathrm{f}(\mathrm{r})$ is the analytical function and $\Gamma$ is the boundary domain of $\Omega$. Separating A into two components of linear and nonlinear terms $\mathrm{L}$ and $\mathrm{N}$ respectively. The Eq. (21) is reconstructed as

$$
L(u)+N(u)-f(r)=0 \quad r \in \Omega
$$

Homotopy perturbation structure takes the form

$$
H(v, p)=(1-p)\left[\mathrm{L}(\mathrm{v})-\mathrm{L}\left(\mathrm{u}_{0}\right)\right]+P[A(v)-f(r)]=0
$$

Where

$$
v(r, p): \Omega x[0,1]-R
$$

$p \epsilon(0,1)$ is the embedding parameter and $\mathrm{U}_{0}$ is taken as the initial term that satisfies boundary condition. The power series of Eq. (24) can be expressed as:

$$
v=v_{0}+p v_{1}+p^{2} v_{2}+p^{3} v_{3}+\ldots
$$

Most appropriate solution for the problem takes the form

$$
u=\lim _{p \rightarrow 1}\left(v_{0}+p v_{1}+p^{2} v_{2}+p^{3} v_{3}+\ldots\right)=v_{0}+v_{1}+v_{2}+v_{3}+\ldots
$$

\section{Application of the homotopy perturbation method to the flow problem}

The homotopy pertubation method which is an analytical scheme for providing approximate solutions to the ordinary differential equations, is adopted in generating solutions to the coupled ordinary nonlinear differential e quation .Upon constructing the homotopy, the Eqs. (11)- (12) can be expressed as

$$
(1-p)\left(f^{i v}\right)+p\left(\begin{array}{l}
f^{i v}-\left(M^{2}+\frac{1}{D a}\right) f^{\prime \prime}+(1-\phi)^{2.5}\left((1-\phi)+\phi \frac{\rho_{s}}{\rho_{f}}\right) R e_{w}\left(f^{\prime \prime \prime}-f f^{\prime \prime \prime}\right) \\
+D e(1-\phi)^{2.5}\left(2 f^{\prime 2} f^{\prime \prime}-2 f f^{\prime \prime 2}+f^{2} f^{i v}\right)=0
\end{array}\right)=0
$$

Taking power series of velocity and rotation fields yields

$$
f=f_{0}+p^{1} f_{1}+p^{2} f_{2}+p^{3} f_{3}+. .
$$

Substituting Eq. (23) into (22) and collecting the like terms of the various order yields

$$
\begin{aligned}
p^{1} & : f_{1}^{i v}-\left(M^{2}+\frac{1}{D a}\right) f_{0}^{\prime \prime}+(1-\phi)^{2.5}\left((1-\phi)+\phi \frac{\rho_{s}}{\rho_{f}}\right) R e_{w}\left(f_{0}^{\prime} f_{0}^{\prime \prime}-f_{0} f_{0}^{\prime \prime \prime}\right) \\
& +D e(1-\phi)^{2.5}\left(2 f_{0}^{\prime 2} f_{0}^{\prime \prime}-2 f_{0} f_{0}^{\prime{ }^{2}}+f_{0}^{2} f_{0}^{i v}\right)=0=0
\end{aligned}
$$

$$
+D e(1-\phi)^{2.5}\left(2{f^{\prime}}^{2} f_{1}^{\prime \prime}-2 f_{1} f_{0}^{\prime 2}+f_{0}^{2} f_{1}^{i v}\right)=0=0
$$

The boundary conditions are

$\eta=0: f_{0}=f_{1}=f_{2}=\ldots=0, \quad f_{0}^{\prime \prime}=f_{1}^{\prime \prime}=f_{2}^{\prime \prime}=\ldots=0$

$$
f_{0}(\eta)=-\frac{1}{2(3 k+1)} \eta^{3}+\frac{3(2 k+1)}{2(3 k+1)} \eta
$$

$\eta=1: f_{0}=f_{1}=f_{2}=\ldots=1, \quad f_{0}^{\prime}=-k f_{0}^{\prime \prime}, f_{1}^{\prime}=-k f_{1}^{\prime \prime}, f_{2}^{\prime}=-k f_{2}^{\prime \prime}, \ldots$

Also, solving Eq. (25) applying the corresponding boundary

On solving Eq. (24) applying the boundary conditions yields conditions yields 


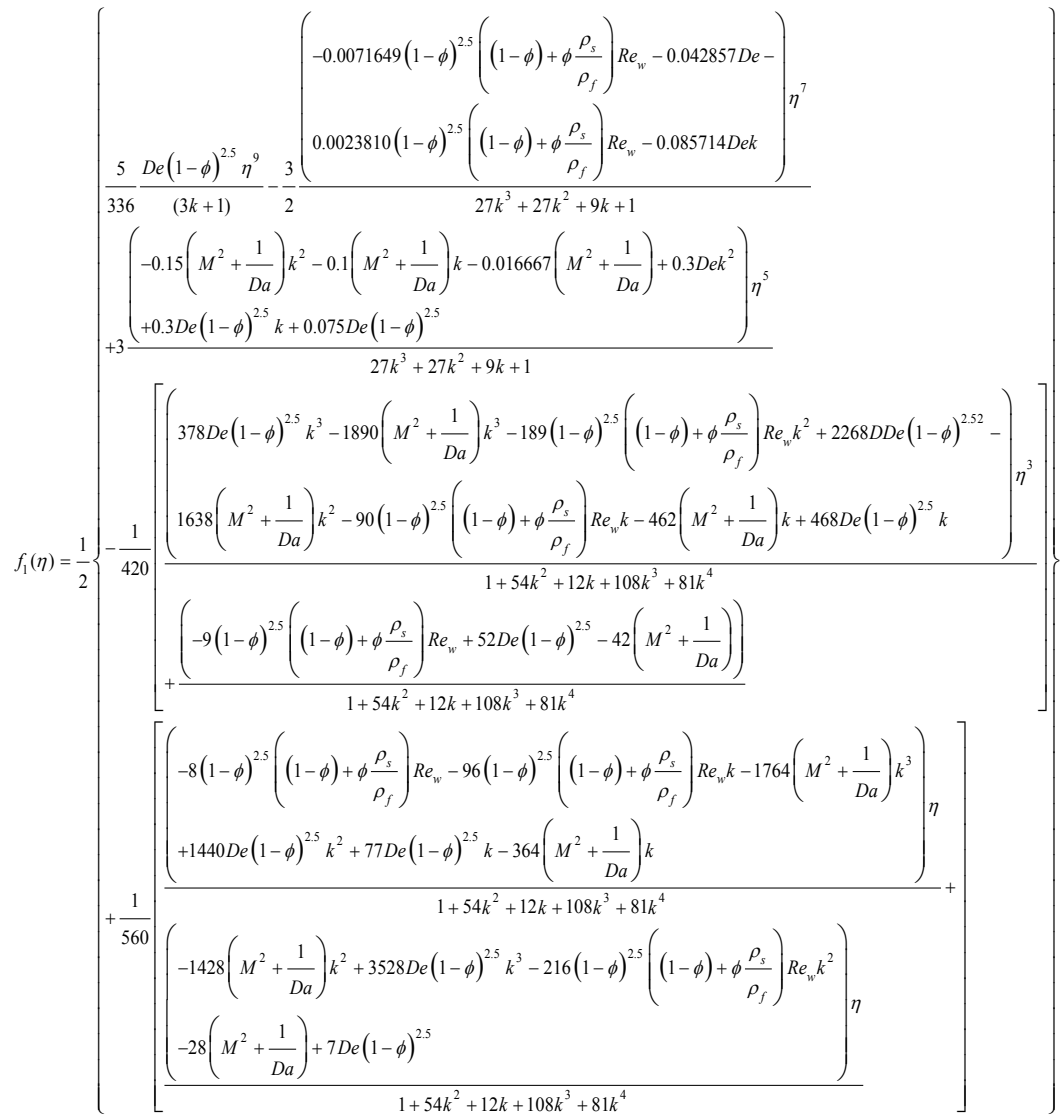

By the definition of homotopy perturbation method, we have

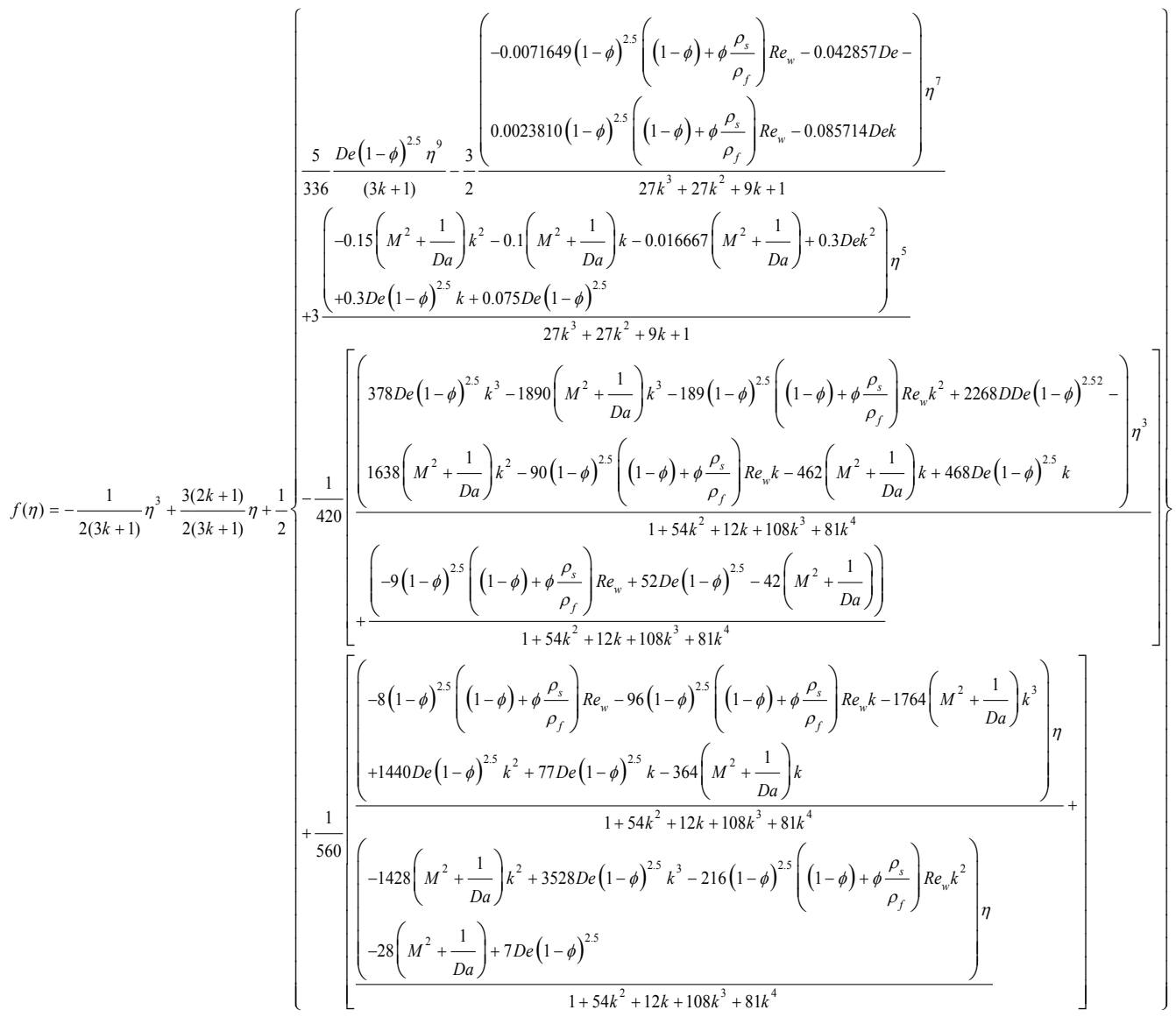




\section{Results and discussion}

The results of the homotopy perturbation method for the problem investigated have been with the results of the fourth-order Runge-Kutta Fehlberg numerical method (for the simplified case) as shown in Table 3. As observed from the Table, good agreements are established between the results of the numerical and homotopy analysis methods. Using copper nanoparticle and water, the results obtained from the analytical solution are shown graphically in Figures 2-9, when $\mathrm{Re}_{\mathrm{w}}=8, \mathrm{De}=0.1, \mathrm{M}=2, \hbar=0.1, \mathrm{Da}=2$ and $\phi=0.01$, unless otherwise stated. Figure 2 depicts the influence of nanoparticle concentration $(\phi)$ on the flow process. As shown from the Figure, as the nanoparticle concentration increases, there is an increase in the velocity distribution. It is very important to indicate viscoelastic nature of the fluid. Therefore, the effects of Deborah's number on the flow process are depicted in Figure 3. In the Figure, it illustrated in that increase in Deborah's number (De) which illustrates the UCM as highly elastic fluid (such as polymeric melts) depicts decrease in fluid flow velocity. The influence of magnetic field parameter on flow of the UCM fluid under is depicted in Figure 4. As observed in the figure, the numerical increase of the magnetic or Hartmann parameter (M) shows decreasing velocity profile. This is because the applied magnetic field produces a damping effect (Lorentz force) on the flow process. This damping affects increases as the quantitative or numerical value of the Hartmann number increases. It should be noted that the effects magnetic field parameter is maximum towards the upper flow channel. In order to shown the effect of the permeability of the porous medium on the flow, effect of Darcy parameter (Da) on fluid transport is illustrated in Figure 5. Increasing Darcy number demonstrates increasing velocity profile as shown in the figure.

Table 3 Comparison of results of numerical and homotopy analysis method for $\mathrm{f}(\eta)$, when $\mathrm{De}=0 . \mathrm{I}, \mathrm{Da}^{-1}=\phi=\mathrm{M}=0, \mathrm{~K}=0 . \mathrm{I}, \mathrm{Re}_{\mathrm{w}}=4$

\begin{tabular}{llll}
\hline \multicolumn{1}{c}{$\boldsymbol{\eta}$} & \multicolumn{1}{c}{ RKFNM } & \multicolumn{1}{c}{ HPM } & |RKFNM-HPM| \\
\hline 0 & 0 & 0 & 0 \\
0.05 & 0.070154 & 0.0701 & $5.46 \mathrm{E}-05$ \\
0.1 & 0.139997 & 0.139899 & $9.76 \mathrm{E}-05$ \\
0.15 & 0.209217 & 0.209199 & $1.77 \mathrm{E}-05$ \\
0.2 & 0.259219 & 0.259201 & $1.76 \mathrm{E}-05$ \\
0.25 & 0.344546 & 0.344499 & $4.76 \mathrm{E}-05$ \\
0.3 & 0.410038 & 0.409999 & $3.96 \mathrm{E}-05$ \\
0.35 & 0.473672 & 0.473598 & $7.37 \mathrm{E}-05$ \\
0.4 & 0.535148 & 0.535102 & $4.59 \mathrm{E}-05$ \\
0.45 & 0.594153 & 0.594098 & $5.51 \mathrm{E}-05$ \\
0.5 & 0.650402 & 0.650348 & $5.37 \mathrm{E}-05$ \\
0.55 & 0.7036 & 0.703084 & $5.16 \mathrm{E}-05$ \\
0.6 & 0.75345 & 0.753398 & $5.19 \mathrm{E}-05$ \\
0.65 & 0.79968 & 0.799599 & $8.18 \mathrm{E}-05$ \\
0.7 & 0.842013 & 0.841977 & $3.56 \mathrm{E}-05$ \\
0.75 & 0.880181 & 0.88013 & $5.06 \mathrm{E}-05$ \\
0.8 & 0.913929 & 0.913899 & $2.94 \mathrm{E}-05$ \\
0.85 & 0.94301 & 0.942976 & $3.4 \mathrm{E}-05$ \\
0.9 & 0.967193 & 0.967159 & $3.45 \mathrm{E}-05$ \\
0.95 & 0.986257 & 0.9862 & $5.74 \mathrm{E}-05$ \\
\hline & 1 & 1 & 0 \\
\hline & & & \\
\hline
\end{tabular}

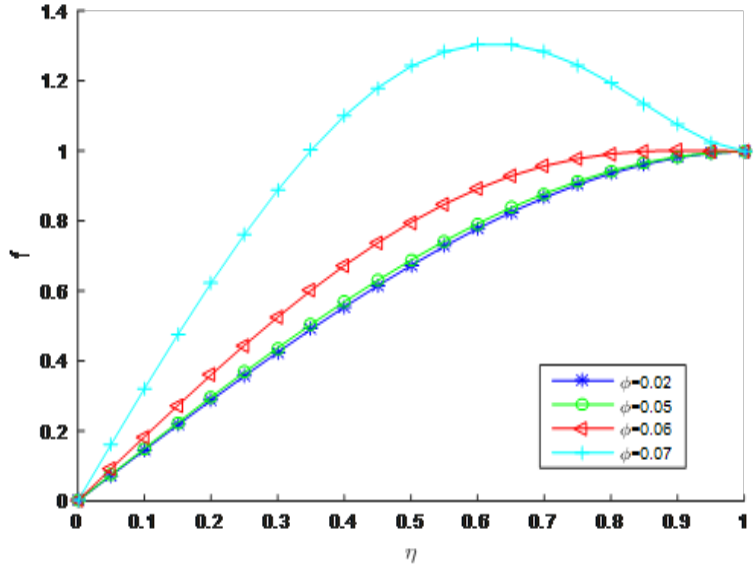

Figure 2 Effecct of nanoparticle concentration number $(\phi)$ on the axial velocity of the flow process.

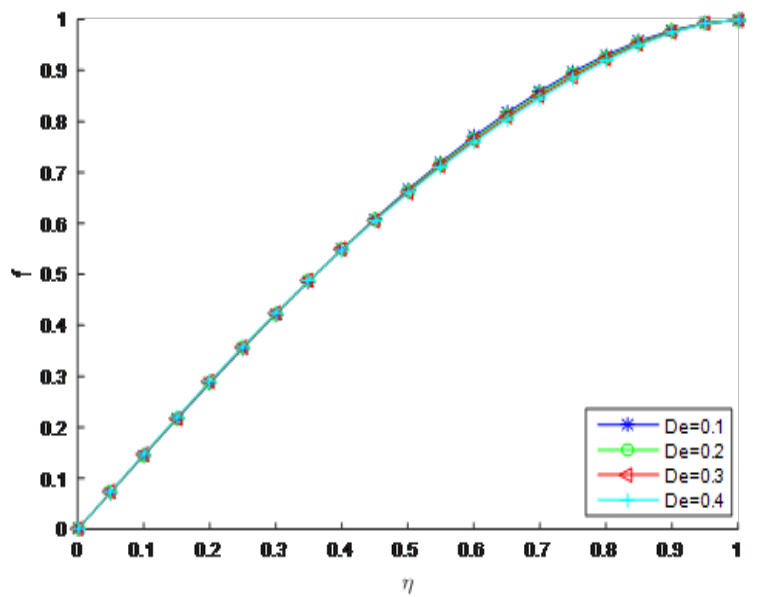

Figure 3 Effect of Deborah's number (De) on the axial velocity of the flow process.

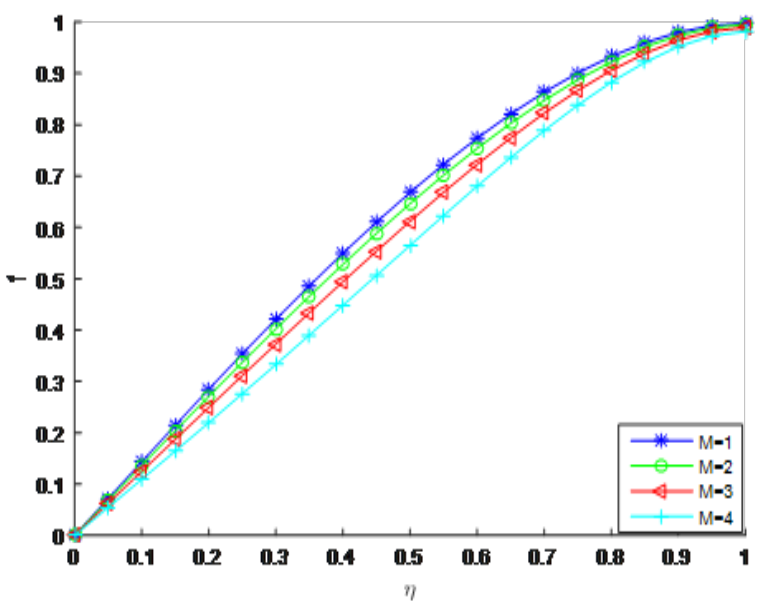

Figure 4 Effect of Hartmann parameter (M) on the axial velocity of the flow process. 


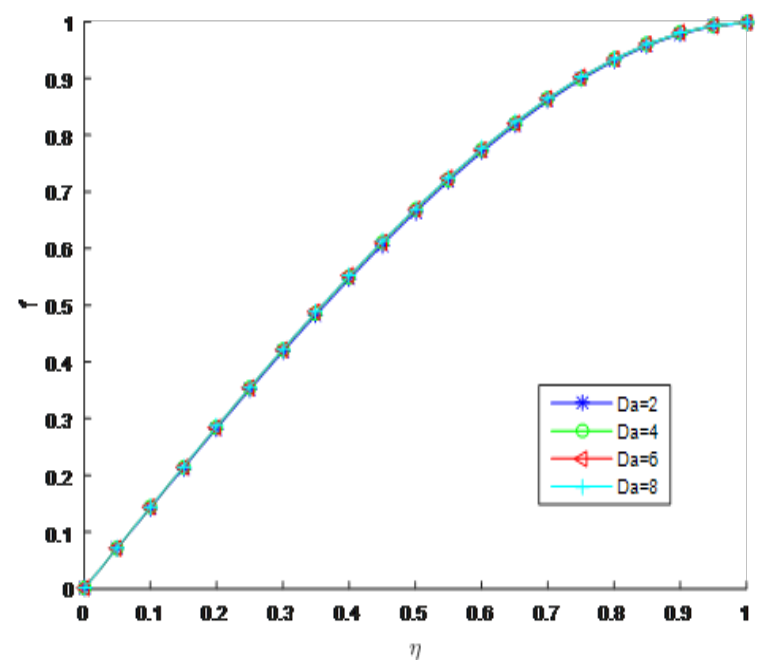

Figure 5 Effect of Darcy's number $(\mathrm{Da})$ on the axial velocity of the flow process.

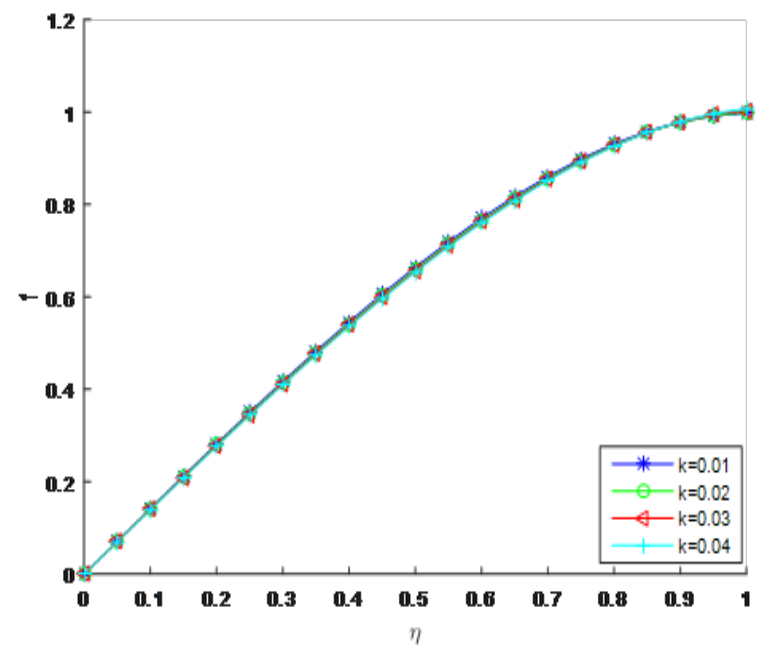

Figure 6 Effect of slip parameter $(k)$ on the axial velocity of the flow process.

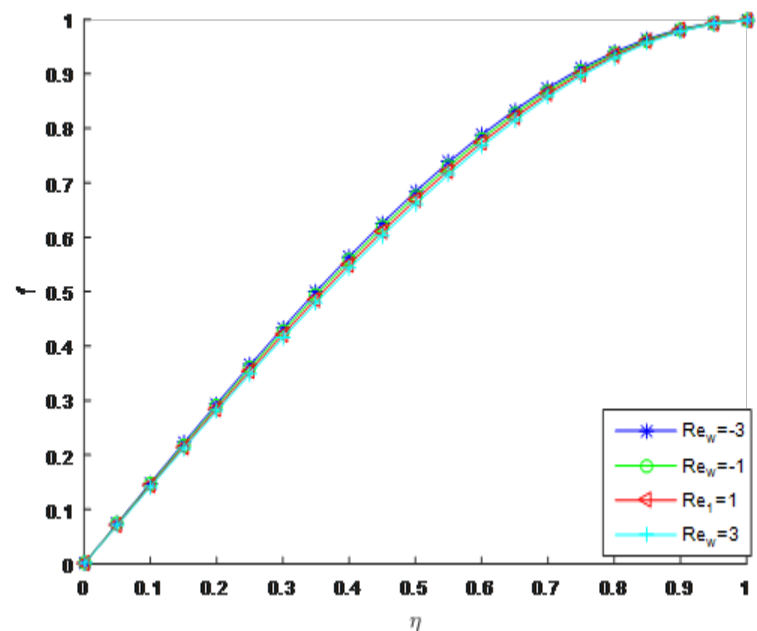

Figure 7 Effect of Reynold's number $\left(\mathrm{Re}_{\mathrm{w}}\right)$ on the axial velocity of the flow process.

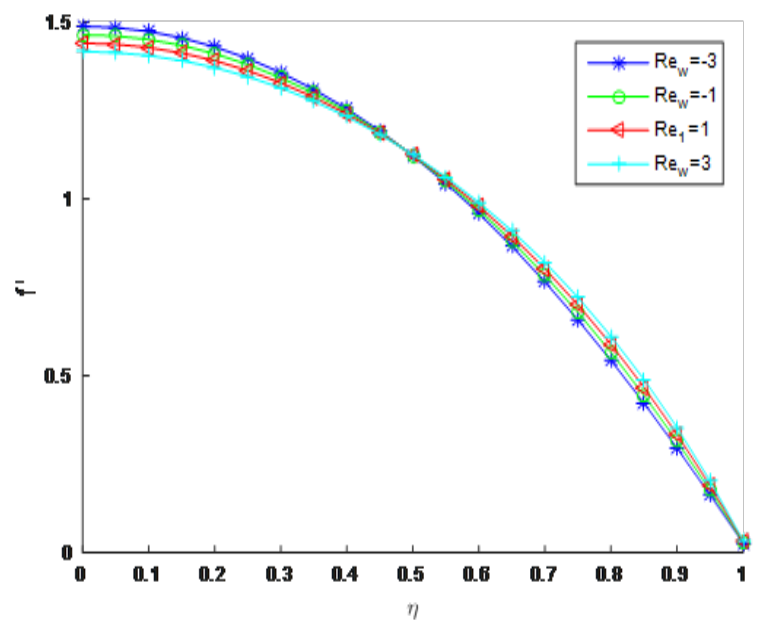

Figure 8 Effect of Reynold's number $\left(\mathrm{Re}_{\mathrm{w}}\right)$ on the radial velocity of the flow process

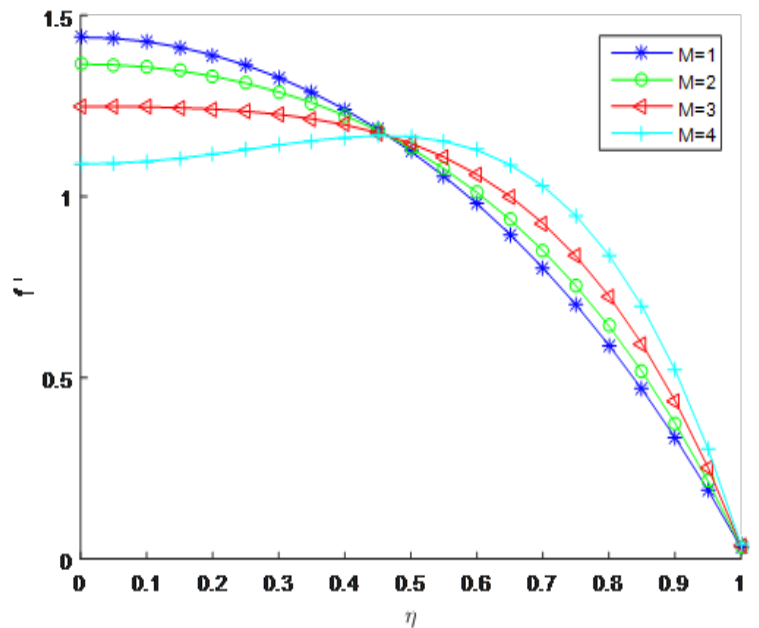

Figure 9 Effect of Hartman parameter $(M)$ on the radial velocity of the flow process.

Figure 6 shows the effect of fluid slip parameter $(\mathrm{k})$ on the velocity of the fluid flow. It should be noted that the slip parameter depicts that the fluid velocity at the boundary is not at equal velocity with fluid particles closest to flow boundary due to large variance in macro and micro fluid flow. As observed from the Figure 6, increasing the slip parameter leads to decreasing velocity distributions of the process. In order to show the relative significance of the inertia effect as compared to the viscous effect, the effect of Reynolds number on the flow phenomena is illustrated in the Figure 7. It is established form the graphical display that increasing Reynolds number $\left(\mathrm{Re}_{\mathrm{w}}\right)$ causes decrease in flow profile which effect is maximum towards the upper plate. Figure 8 shows the effect of Reynolds number on the radial velocity component of the flow. It is shown that increasing the Reynolds number causes decrease in velocity distribution but as flow reaches the mid plate around $\eta=0.5$ (not determined accurately) an increasing velocity distribution is seen. However, effect is minimal towards the upper plate. Also, influence of magnetic field on radial velocity is depicted in Figure 9, as shown significant increase in velocity is seen due to quantitative increase of Hartmann parameter (M) towards the lower plate while as upper plate is approached a reverse trend is observed. 


\section{Conclusion}

In this work, homotopy perturbation method is used to analyze the flow of an upper convective Maxwell (UCM) nanofluid through a permeable microchannel embedded in a porous medium and under the influence of slip condition has been presented. Important fluid parameter effect such as Deborah's number, Darcy parameter and Hartman parameter on the fluid flow was investigated. that increase in slip parameter, nanoparticle concentration and Darcy number lead to increase in the velocity of the upper-convected Maxwell fluid while increase in Deborah's, Hartmann and Reynold numbers decrease the fluid flow velocity towards the lower plate but as the upper plate is approached, a reverse trend is observed. The results obtained in this work can be used to further the applications of UCM fluid in biomedical, astrophysics, geosciences etc.

\section{Acknowledgements}

None.

\section{Conflict of interest}

The authors declare that there is no conflict of interest.

\section{Nomenclature}

$\begin{array}{ll}\mathrm{Re}_{w} & \text { Reynolds number } \\ \mathrm{M} & \text { Hartman parameter } \\ \mathrm{K} & \text { Slip parameter } \\ \mathrm{HAM} & \text { Deborah's number } \\ \hbar & \text { Homotopy analysis method } \\ v^{*} & \text { Auxilliary parameter } \\ u^{*} & \text { y axis velocity component } \\ x & \text { x axis velocity component } \\ y & \text { Dimensionless horizontal coordinate } \\ x^{*} & \text { Dimensionless vertical coordinate } \\ y^{*} & \text { Distance in x axis parallel to plate } \\ \text { Da } & \text { Distance in y axis parallel to plate } \\ & \text { Darcy number }\end{array}$

\section{Symbols}

$\begin{array}{ll}\rho & \text { Fluid density } \\ \lambda & \text { Relaxation time } \\ v & \text { Kinematic viscosity } \\ \beta & \text { Sliding friction } \\ \phi & \text { Nanoparticle concentration }\end{array}$

\section{References}

1. Bird RB, Armstrong RC, Hassager O. Dynamics of Polymeric liquids. New York: Wiley; 1987.

2. Sadeghy K, Najafi AH, Saffaripour M. Sakiadis flow of an upperconvected Maxwell fluid. International Journal of Non-Linear Mechanics. 2005;40(9):1220-1228.
3. Ziabaksh Z, Domairry G. Solution of laminar viscous flow in semi porous channel in the presence of a uniform magnetic field by using homotopy analysis method. Communications in nonlinear science and numerical simulations. 2009;14(4):1284-1294.

4. Fetecau C. A new exact solution for the flow of a Maxwell fluid past an infinite plate. International Journal of Non-Linear Mechanics. 2003;38(3):423-427.

5. Hunt JCR. Magnetohydrodynamic flow in rectangular ducts. Journal of Fluid Mechanics. 1965;21(4):577-590.

6. Sheikholeslami M, Hatami M, Ganji DD. Analytical investigation of MHD nanofluid flow in a semi-porous channel. Powder Technology. 2013;246:327-336.

7. Sheikholeslami M, Gorji-Bandpy M, Ganji DD. Numerical investigation of $\mathrm{MHD}$ effects on $\mathrm{Al}_{2} \mathrm{O}_{3}$-water nanofluid flow and heat transfer in a semiannulus using LBM. Energy. 2013;60:501-510.

8. Sheikholeslami M, Gorji-Bandpy M, Ganji DD. Lattice Boltzman method for MHD natural convection heat transfer using nanofluid. Powder Technology. 2014;254:82-93.

9. Sheikholeslami M, Hatami M, Ganji DD. Nano fluid flow and heat transfer in a rotating system in the presence of a magnetic field. Journal of Molecular Liquids. 2014;190:112-120.

10. Raftari B, Yildirim A. The application of homotopy perturbation method for MHD flows above porous stretching sheets. Computers \& Mathematics with Applications. 2010;59(10):740-744.

11. Sheikholeslami M, Ganji DD. Entropy generation of nanofluid in presence of magnetic field using lattice boltzmann method. Physica A. 2015;417:273-286.

12. Ganji DD, Kachapi H, Seyed H. Analytical and numerical method in engineering and applied science. Progress Nonlinear Science. 2011;3:570 579 .

13. Sadeghy K, Sharifi M. Local similarity solution for the flow of a "second grade" viscoelastic fluid above a moving plate. International Journal of Non-Linear Mechanics. 2004;39(8):1265-1273.

14. Vajrevulu K, Prasad KV, Sujatha A. MHD flow and mass transfer of chemically reactive upper convected Maxwell fluid past porous surface. Applied Mathematical Mechanics. 2012;33(7):899-910.

15. Raftari B, Vajrevulu K. Homotopy analysis method for MHD viscoelastic fluid flow and heat transfer in a channel with a stretching wall. Communications in Nonlinear Science and Numerical Simulation. 2012;17(11):4149-4162.

16. Hatami M, Nouri R, Ganji DD. Forced convection analysis for MHD $\mathrm{Al}_{2} \mathrm{O}_{3}$-water nanofluid flow over a horizontal plate. Journal of Molecular Liquids. 2013;187:294-301.

17. Aziz A. A similarity solution for laminar thermal boundary layer over a flat plat with a convective surface boundary condition. Communication in Nonlinear Science and Numerical Simulation. 2009;14(4):1064-1068.

18. Beg OA, Makinde OD. Viscoelastic flow and species transfer in a Darcian high permeability channel. Journal of Petrol Science Engineering. 2011;76(3-4):93-99.

19. Navier CLMH. Memoir sur les lois du movement des fluids. Memoirs Academic Royal Science Institute France; 1823. 389-440 p.

20. Choi CH, Westin JA, Breur KS. To slip or not slip water flows in hydrophilic and hydrophobic microchannels. New Orleans: Proceedings of IMECE; 2002.

21. Matthew MT, Hill JM. Nano boundary layer equation with nonlinear Navier boundary condition. Journal of Mathematical Analysis and Application. 2007;333(1):381-400. 
22. Martin MJ, Boyd LD. Momentum and heat transfer in a laminar boundary layer with slip flow. Journal of Thermo physics and Heat Transfer. 2006;20(4):710-719.

23. Ariel PD. Axisymmetric flow due to stretching sheet with partial slip. Computer and Mathematics with Application. 2007;54(7-8):381-400.

24. Wang C. Analysis of viscous flow due to a stretching sheet with surface slip and suction. Nonlinear Analysis: Real world applications. 2009;10(1):375-380.

25. Das K. Impact of thermal radiation on MHD slip flow over a flat plate with variable fluid properties. Heat and mass transfer. 2012;48(5):767-778.

26. Das K. Slip effects on heat and mass transfer in MHD micro polar fluid flow over an inclined plate with thermal radiation and chemical reaction. International Journal of Numerical methods in Fluids. 2016;70(1):96-101.

27. Hussain A, Mohyud-Cheema ST, Din TA. Analytical and numerica approaches to squeezing flow and heat transfer between two paralle disks with velocity slip and temperature jump. China Physics Letters. 2012;29(11):1-5.

28. Das K. Slip flow and convective heat transfer of nanofluid over a permeable stretching surface. Computers \& Fluids. 2012;64(1):34-42.

29. Deissler RG. An analysis of second-order slip flow and temperature-jump boundary conditions for rarefied gases. International Journal of heat and mass transfer. 1964;7(6):681-694
30. Das K, Jana S, Acharya N. Slip effects on squeezing flow of nanofluid between two parallel disks. International Journal of Applied Mechanics and Engineering. 2016;21(1):5-20.

31. Ellahi R, Hayat T, Mahomed FM, et al. Effects of slip on nonlinear flows of a third grade fluid. Nonlinear Analysis: Real world Applications. 2010;11(1):139-146.

32. Malvadi A, Hedayati F, Ganji DD. Slip effects on unsteady stagnation point flow of a nanofluid over a stretching sheet. Powder Technology. 2014;253:377-384.

33. Zhang Y, Zhang M, Bai Y. Unsteady flow and heat transfer of power-law nanofluid thin film over a stretching sheet with variable magnetic field and power-law velocity slip effect. J Taiwan Inst Chem Eng. 2017;70:104-110.

34. Zhang Y, Zhang M, Bai Y. Flow and heat transfer of an Oldroyd-B nanofluid thin film over an unsteady stretching sheet. J Mol Liq. 2016;220:665-670.

35. Zhang Y, Zhang M, Bai Y. Unsteady Cattaneo-Christov double diffusion of Oldroyd-B fluid thin film with relaxation-retardation viscous dissipation and relaxation chemical reaction. Powder Technol. 2018;338:975-982.

36. Abbasi M, Khaki M, Rahbari A, et al. Analysis of MHD flow characteristics of an UCM viscoelastic flow in a permeable channel under slip conditions. Journal of Brazilian Society of Mechanical Engineering Science. 2016;38(3):977-988. 